\title{
Extremum Seeking Control of a Nonholonomic System with Sensor Constraints *
}

\author{
Yinghua Zhang ${ }^{\mathrm{a}}$, Oleg Makarenkov ${ }^{\mathrm{b}}$, Nicholas Gans ${ }^{\mathrm{a}}$,

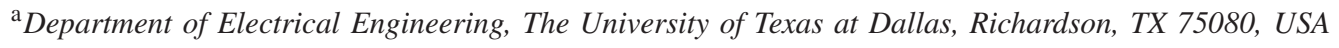 \\ ${ }^{\mathrm{b}}$ Department of Mathematical Sciences, The University of Texas at Dallas, Richardson, TX 75080, USA
}

\begin{abstract}
This paper presents an extremum seeking control (ESC) scheme for mobile robots with nonholonomic constraints. Many sensors, such cameras, have a limited field of view. If a target function is based on these sensors, correct robot orientation is critical for maximizing the target function. Our approach is novel in that it will maximize a target function that is a function of robot position and orientation, while overcoming the nonholonomic constraints that prevent simple motion along the gradient of all degrees of freedom. Stability analysis proves our ESC scheme is well behaved and the robot will settle in the neighborhood of a maximum of the target function. Simulation and experiment results elucidate performance for different tasks.
\end{abstract}

Key words: mobile robot; optimization; non-holonomic constraints.

\section{Introduction}

Mobile robots can be used for many automatic searching tasks, such as seeking resources, search and rescue, surveillance, and exploration. Many of these tasks can be modeled as an optimization problem. The search area is the feasible range of the problem, and the mobile robot acts as an observer, measuring some physical variable related with the searching task. The measurement at every point in the feasible range constitutes a measurement-to-point mapping or a target function. The mobile robot then seeks the point where the measurement is at its maximum. For example, in the source seeking task, a mobile robot is equipped with a sensor to measure the strength of a scalar signal emitted by a source and seeks a path to this source $[1,14,16,25]$.

In many cases there is little or no a priori information of the target function, nor is there absolute information of the the robot's location. In these situations, extremum seeking control (ESC) algorithms are a strong option $[1-3,5,7,11$,

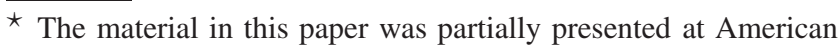
Control Conference, Washington D.C., Jun, 2013 [26]. This work was supported in part by DGIST Program of the Ministry of Education, Science and Technology of Korea (12-BD-0101) and by NSF Grant CMMI-1436856. Corresponding author Nicholas Gans. Tel. +1-972-883-4341

Email addresses: yxz102220@utdallas.edu (Yinghua Zhang), oxm130230@utdallas.edu (Oleg Makarenkov), ngans@utdallas.edu (Nicholas Gans).
}

12, 14, 16-22, 25]. Many of ESC algorithms attempt to find the maximum value of the target function by adjusting the control inputs according to a gradient estimation algorithm implemented in real time. The gradient is estimated by using an external, periodic perturbation and a series of filtering and modulation operations. The estimated gradient is integrated to generate the control inputs, which constitute an estimate of the optimal set of variables. If the ESC loop is stable, the inputs to the integrators will vanish when the system reaches steady state. This results in a zero gradient, which is a necessary condition for unconstrained optimization. Thus, in steady state, the system is at a local maximum.

For nonholonomic mobile robots, the ESC problem is particularly difficult, as nonholonomic constraints prevent the system from actuating along all degrees of freedom. It is therefore impossible to move along an arbitrary gradient. Unique ESC algorithms were presented in [1, 6, 14, 16, 25] to search for the maximum of a scalar measurement in a $2 \mathrm{D}$ plane. In these works, the functions being maximized were functions of position, but not orientation. That is, the maximum of the target function is invariant to robot orientation.

When the target function is dependent on the robot orientation, the problem becomes more challenging. A constant angular velocity, as used in [25], may no longer be applicable, as the robot cannot settle at an orientation that will maximize the signal. On the other hand, if a robot keeps a constant linear velocity as in $[1,14,16]$, the robot must maintain a curved 
trajectory in which the orientation sweeps through all values (e.g. circle or rose curve) to stay in a bounded neighborhood around the maximum point. Thus the robot can not settle at any fixed orientation. In the case that the angular velocity is tuned by ESC and the linear velocity is regulated by a derivative control law as in [6], the robot is able to settle at a fixed orientation, but this settled orientation is somewhat arbitrary and has no relation with the target function. The problem becomes more difficult still for robots with sensors that have limited field of view (FOV), since the robot has to keep its target in the FOV [4] or it will lose the measurement. Examples of such kind of tasks that depend on orientation and FOV include mobile robots equipped with forward facing camera, marine vehicles equipped with active sonar, or vehicles equipped with active radar or lidar.

In this paper, we propose an ESC scheme for nonholonomic mobile robots in $\mathrm{SE}(2)$, that is, optimal pose seeking including both the optimal position and optimal orientation. We are motivated by tasks in which a robot equipped with a camera seeks to maximize the value of the image information in the current view $[23,24]$. A related problem would be searching for a target via template matching, in which a mobile robot equipped with a camera continuously computes the correlation function between the current image and a preloaded image and moves to a position where the correlation function achieves its maximum. Another motivating problem is a mobile robot seeking to maximize communication signal strength by properly aiming its antenna.

In the following sections, we first introduce the model of nonholonomic mobile robot, and the definition of averaged system. Then, the proposed SE(2) ESC algorithm is presented in detail, and its stability is proved under the assumption of quadratic target function. In the end, simulations and experiments are shown to demonstrate its performance.

\section{Background}

\subsection{Nonholonomic Mobile Robots}

We adopt the kinematic unicycle model for our nonholonomic mobile robot. The coordinates of the robot state are $[x, y, \theta]^{T}$, where $x$ and $y$ are the position coordinates of the robot in a $2 \mathrm{D}$ plane and $\theta$ is the orientation angle. The equations of state can be written in matrix form as

$$
\left[\begin{array}{c}
\dot{x} \\
\dot{y} \\
\dot{\theta}
\end{array}\right]=\left[\begin{array}{c}
\cos (\theta) \\
\sin (\theta) \\
0
\end{array}\right] v+\left[\begin{array}{l}
0 \\
0 \\
1
\end{array}\right] w
$$

where $v$ and $w$ are linear and angular velocity, respectively, and are inputs to the system.

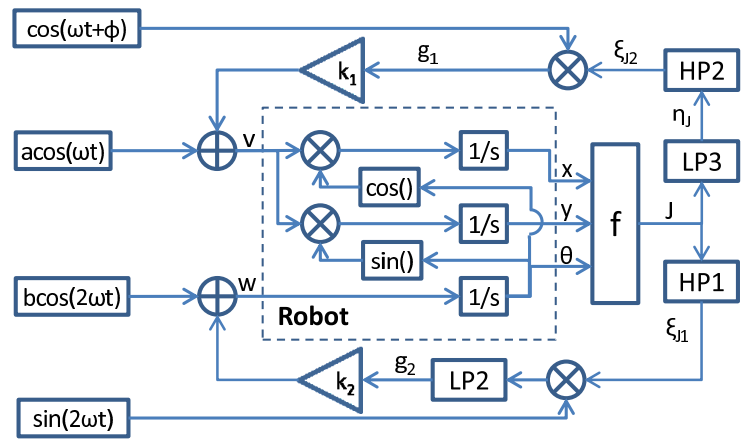

Fig. 1. Our proposed ESC scheme for mobile robots

\subsection{Averaged System}

If a system is given by $\dot{\mathbf{x}}=\varepsilon \mathbf{f}(\mathbf{x}, t, \varepsilon)$, where $\mathbf{x} \in \mathbb{R}^{n}, \varepsilon$ is a small positive parameter, and $\mathbf{f}(\mathbf{x}, t, \varepsilon)$ is $T$-periodic in $t$, the averaged system is

$$
\dot{\mathbf{x}}^{a}=\varepsilon \overline{\mathbf{f}}\left(\mathbf{x}^{a}\right)
$$

where $\overline{\mathbf{f}}\left(\mathbf{x}^{a}\right)=\frac{1}{T} \int_{0}^{T} \mathbf{f}\left(\mathbf{x}^{a}, t, 0\right) d t$. See [10] for details of averaging theory and methods.

\section{System Model}

The proposed ESC scheme for nonholonomic mobile robot is shown in Fig. 1. The target function we are going to explore is represented by the block $\mathrm{f}$, and the function value $J=f(x, y, \theta)$. A group of linear filters are used in this scheme: the low pass filters LP2, LP3 and high pass filters HP1, HP2. We implemented them as 1st order filters. The transfer functions of LP2 and LP3 are respectively $\frac{\omega_{2}}{s+\omega_{2}}$ and $\frac{s\left(1-k_{3}\right)+\omega_{1}}{s+\omega_{1}}$, and the transfer function of HP1 and HP2 are respectively $\frac{s}{s+\omega_{1}}$ and $\frac{s}{s+\omega_{2}}$, where the $\omega_{1}$ and $\omega_{2}$ are cutoff frequencies for the filters, and the constant $k_{3} \neq 1$. The signals $\eta_{J}, \xi_{J 2}$ and $\xi_{J 1}$ are outputs of LP3, HP2 and HP1 respectively. The inputs $a \cos (\omega t)$ and $b \cos (2 \omega t)$ serve as dithers for $v$ and $w$ respectively, and $\cos (\omega t+\phi)$ and $\sin (2 \omega t)$ serve as demodulating signals, where the phase $\phi$ is constant. The signals $g_{1}$ and $g_{2}$ are the signals of estimated gradients of the target function with respect to position and orientation. They are multiplied by constants $k_{1}$ and $k_{2}$ before being applied as input to the robot in (1).

In contrast with typical multi-dimensional ESC, an additional low-pass filter LP3 is necessary in the linear velocity feedback loop. The linear and angular velocity inputs have different dither frequencies. LP3 discriminates the components from each input in the output signal to facilitate the demodulation and estimation process. Considering the system bandwidth, the frequency difference cannot be too large. Thus, we suggest $2 \omega$ for the angular dither frequency.

To facilitate the proof of system stability, we transform the system block diagram to an equivalent form shown in Fig. 2 , in which the target function $f$, dither signals, and constant 


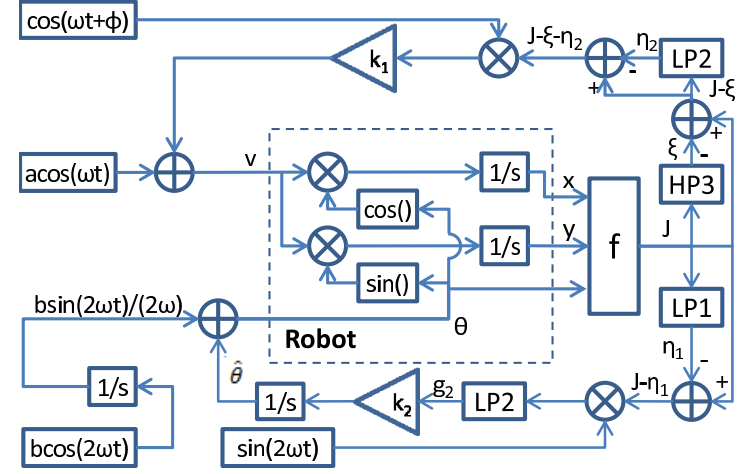

Fig. 2. The equivalent ESC scheme for stability analysis

gains are same as Fig. 1. The first difference is that we transform LP3 in Fig. 1 to the form of an input minus a high pass filter. Define $\xi$ as the outputs of HP3

$$
\dot{\xi}=-\omega_{1} \xi+k_{3} \dot{J}
$$

The second difference is that we transform the two high pass filters in Fig. 1 to the form of input minus a low pass filter. Define $\eta_{1}, \eta_{2}$ as the outputs of LP1 and the LP2 in the lower branch respectively

$$
\begin{aligned}
& \dot{\eta_{1}}=-\omega_{1} \eta_{1}+\omega_{1} J \\
& \dot{\eta}_{2}=-\omega_{2} \eta_{2}+\omega_{2}(J-\xi) .
\end{aligned}
$$

As illustrated in Fig. 2, the robot position dynamics are

$\dot{x}=\cos (\theta)\left[a \cos (\omega t)+k_{1}\left(J-\xi-\eta_{2}\right) \cos (\omega t+\phi)\right]$

$\dot{y}=\sin (\theta)\left[a \cos (\omega t)+k_{1}\left(J-\xi-\eta_{2}\right) \cos (\omega t+\phi)\right]$.

and the robot orientation coordinates satisfy

$\ddot{\hat{\theta}}=-\omega_{2} \dot{\hat{\theta}}+\omega_{2} k_{2}\left(J-\eta_{1}\right) \cos (2 \omega t)$

$\theta=\frac{1}{2 \omega} b \sin (2 \omega t)+\hat{\theta}$.

As a benchmark example of ESC, we assume that the target function is quadratic ${ }^{1}$ and in the form

$$
J=f^{*}-k_{p}\left(x-x^{*}\right)^{2}-k_{p}\left(y-y^{*}\right)^{2}-k_{o}\left(\theta-\theta^{*}\right)^{2}
$$

where $k_{p}$ and $k_{o}$ are positive constants, and $\left[x^{*}, y^{*}, \theta^{*}\right]^{T}$ is the maximum point.

1 The quadratic assumption is typical when proving stability of ESC systems $[6,12,13,20]$. This is justified by the fact that analytic target functions can be locally expanded to a Taylor series with respect to the variable of dither amplitude. We prove stability under the conditions of quadratic target function, but show through experiments that the optimization method works well even when the objective function is much more complicated than a quadratic model, including having local optima.

\section{Stability Analysis}

The stability will be established via the method of averaging with $k_{1}, \omega_{1}, k_{2}, \omega_{2}$ playing the role of small parameters. To transform system detailed in (2)-(5) to the standard form of averaging, we solve (2)-(5) with $k_{1}=\omega_{1}=k_{2}=\omega_{2}=0$ and define a new set of coordinates

$$
\begin{aligned}
& \tilde{\theta}(t)=\theta(t)-\theta^{*}-\frac{b}{2 \omega} \sin (2 \omega t) \\
& \tilde{x}(t)=x(t)-x^{*}-a \int_{0}^{t} \cos (\theta(\tau)) \cos (\omega \tau) d \tau \\
& \tilde{y}(t)=y(t)-y^{*}-a \int_{0}^{t} \sin (\theta(\tau)) \cos (\omega \tau) d \tau \\
& \tilde{\xi}(t)=\xi(t)-k_{3} J .
\end{aligned}
$$

Defining $k_{1}=\delta k_{1}^{\prime}, \omega_{1}=\delta, \omega_{1}^{\prime}, k_{2}=\delta k_{2}^{\prime}, \omega_{2}=\delta \omega_{2}^{\prime}$, where $\delta$ is a small positive parameter, and introducing $\alpha=\frac{1}{\omega_{2}} \dot{\tilde{\theta}}$, system (2)-(5) takes the form

$$
\begin{aligned}
\dot{\tilde{\theta}} & =\delta \omega_{2}^{\prime} \alpha, \\
\dot{\alpha} & =-\delta \omega_{2}^{\prime} \alpha+\delta k_{2}^{\prime}\left(J-\eta_{1}\right) \sin (2 \omega t), \\
\dot{\tilde{x}} & =\delta g_{c s}(t) a k_{1}^{\prime}\left(J-k_{3} J-\tilde{\xi}-\eta_{2}\right), \\
\dot{\tilde{y}} & =\delta g_{s s}(t) a k_{1}^{\prime}\left(J-k_{3} J-\tilde{\xi}-\eta_{2}\right), \\
\dot{\eta}_{1} & =-\delta \omega_{1}^{\prime} \eta_{1}+\delta \omega_{1}^{\prime} J \\
\dot{\eta}_{2} & =-\delta \omega_{2}^{\prime} \eta_{2}+\delta \omega_{2}^{\prime}\left(J-k_{3} J-\tilde{\xi}\right), \\
\dot{\tilde{\xi}} & =-\delta \omega_{1}^{\prime}\left(\tilde{\xi}+k_{3} J\right)
\end{aligned}
$$

where

$$
\begin{aligned}
& J= f^{*}-k_{p}\left(\tilde{x}+a g_{c}(t)\right)^{2}-k_{p}\left(\tilde{y}+a g_{s}(t)\right)^{2}- \\
&-k_{0}\left(\tilde{\theta}+\frac{b}{2 \omega} \sin 2 \omega t\right)^{2} . \\
& g_{s s}(t)= \sin \left(\tilde{\theta}+\theta^{*}+\frac{b}{2 \omega} \sin (2 \omega t)\right) \cos (\omega t+\phi) \\
& g_{c s}(t)= \cos \left(\tilde{\theta}+\theta^{*}+\frac{b}{2 \omega} \sin (2 \omega t)\right) \cos (\omega t+\phi) \\
& g_{c c}(t)=\cos \left(\tilde{\theta}+\theta^{*}+\frac{b}{2 \omega} \sin (2 \omega t)\right) \cos (\omega t) \\
& g_{s c}(t)=\sin \left(\tilde{\theta}+\theta^{*}+\frac{b}{2 \omega} \sin (2 \omega t)\right) \cos (\omega t) \\
& g_{c}(t)=\int_{0}^{t} g_{c c}(\tau, \theta) d \tau \quad g_{s}(t)=\int_{0}^{t} g_{s c}(\tau, \theta) d \tau .
\end{aligned}
$$

The following theorem provides sufficient conditions the system (7) to admit an locally exponentially stable periodic solution, specifically if $k_{3} \neq 1$ and $\phi \neq 0$.

Theorem. If $k_{3} \neq 1$, then for each small $|\phi|>0$ with

$$
\left(1-k_{3}\right) \phi>0
$$


there exists $b_{0}>0$ such that given any $b \in\left(0, b_{0}\right]$ one can find $a_{0}>0$ such that for all $a \in\left(0, a_{0}\right)$, the following property holds: for any $\delta>0$ sufficiently small, system (7) has a locally exponentially stable $2 \pi / \omega$-periodic solution

$$
\left\|\left(\tilde{\theta}, \alpha, \tilde{x}, \tilde{y}, \eta_{1}, \eta_{2}, \tilde{\xi}\right)(t)^{T}\right\| \leq L\left(a+b^{2}+\delta\right),
$$

$t \geq 0$, where $L>0$ is independent of $a, b$, and $\delta$.

Proof. Step 1. As the first step we average system (7) over the time $[0,2 \pi / \omega]$ (see formulas (10.24)-(10.25) in [10]) and prove the existence of an equilibrium for the averaged system. Since

$$
\begin{aligned}
& \int_{0}^{2 \pi / \omega} \sin (2 \omega t) d t=\int_{0}^{2 \pi / \omega} \sin ^{3}(2 \omega t) d t=0 \\
& \int_{0}^{2 \pi / \omega} \sin (2 \omega t) g_{c}(t) d t=\int_{0}^{2 \pi / \omega} \sin (2 \omega t) g_{s}(t) d t=0
\end{aligned}
$$

for all $\tilde{\theta}$ and $\phi$, averaging the first two equations of (7) over the period $[0,2 \pi / \omega]$ (i.e., integrating over $[0,2 \pi / \omega]$ and dividing by $2 \pi / \omega)$ yields

$$
\left(\begin{array}{c}
\dot{\tilde{\theta}}^{a} \\
\dot{\alpha}^{a}
\end{array}\right)=\delta A_{1}\left(\begin{array}{c}
\tilde{\theta}^{a} \\
\alpha^{a}
\end{array}\right)-\delta h_{1}\left(\tilde{\theta}^{a}\right)
$$

where

$$
\begin{gathered}
A_{1}=\left(\begin{array}{cc}
0 & \omega_{2}^{\prime} \\
-k_{2}^{\prime} k_{0}(b / \omega) & -\omega_{2}^{\prime}
\end{array}\right) \\
h_{1}(\tilde{\theta})=\left(\begin{array}{c}
0 \\
a^{2} k_{2}^{\prime} k_{p} \frac{\omega}{2 \pi} \int_{0}^{2 \pi / \omega} \sin (2 \omega t)\left(g_{c}^{2}(t)+g_{s}^{2}(t)\right) d t
\end{array}\right) .
\end{gathered}
$$

To find an equilibrium of (10), note that $\left(\tilde{\theta}_{0}^{a}, \alpha_{0}^{a}\right)=(0,0)$ when $a k_{2}^{\prime} k_{p}=0$. Since $A_{1}$ is invertible, the Implicit Function Theorem implies that (10) admits an equilibrium

$$
\left\|\left(\tilde{\theta}_{0}^{a}, \alpha_{0}^{a}\right)\right\|=O\left(a^{2} k_{2}^{\prime} k_{p}\right) .
$$

Since

$$
\begin{aligned}
& \int_{0}^{2 \pi / \omega} g_{c s}(t) d t=\int_{0}^{2 \pi / \omega} g_{s s}(t) d t=0 \\
& \int_{0}^{2 \pi / \omega} g_{c s}(t) \sin (2 \omega t) d t=\int_{0}^{2 \pi / \omega} g_{s s}(t) \sin (2 \omega t) d t=0 \\
& \int_{0}^{2 \pi / \omega} g_{c s}(t) \sin ^{2}(2 \omega t) d t=\int_{0}^{2 \pi / \omega} g_{s s}(t) \sin ^{2}(2 \omega t) d t=0
\end{aligned}
$$

for all $\tilde{\theta}$ and $\phi$, averaging the second two equations of (7) over the time interval $[0,2 \pi / \omega]$ gives

$$
\left(\begin{array}{c}
\dot{\tilde{x}}^{a} \\
\dot{\tilde{y}}^{a}
\end{array}\right)=-\delta k_{1}^{\prime}\left(1-k_{3}\right) k_{p} a\left(2 A_{2}\left(\tilde{\theta}^{a}\right)\left(\begin{array}{c}
\tilde{x}^{a} \\
\tilde{y}^{a}
\end{array}\right)+a h_{2}\left(\tilde{\theta}^{a}\right)\right)
$$

where

$$
\begin{aligned}
A_{2}(\tilde{\theta}) & =\frac{\omega}{2 \pi} \int_{0}^{2 \pi / \omega}\left(\begin{array}{ll}
g_{c s}(t) g_{c}(t) & g_{c s}(t) g_{s}(t) \\
g_{s s}(t) g_{c}(t) & g_{s s}(t) g_{s}(t)
\end{array}\right) d t \\
& =:\left(\begin{array}{l}
\overline{g_{c s} g_{c}} \overline{g_{c s} g_{s}} \\
\overline{g_{s s} g_{c}} \overline{g_{s s} g_{s}}
\end{array}\right) \\
h_{2}(\tilde{\theta}) & =\frac{\omega}{2 \pi} \int_{0}^{2 \pi / \omega}\left(\begin{array}{l}
g_{c s}(t) \\
g_{s s}(t)
\end{array}\right)\left(g_{c}^{2}(t)+g_{s}^{2}(t)\right) d t .
\end{aligned}
$$

We need to establish that $\operatorname{det}\left(A_{2}\right)>0$ given that $\phi>0, b>$ 0 are sufficiently small. Define $g(b, \phi)=\operatorname{det}\left(A_{2}\right)$, and let $g_{b}^{\prime}(b, \phi), g_{b b}^{\prime \prime}(b, \phi)$ denote the first and second derivatives of $g(b, \phi)$ with respect to $b$. Taking the Taylor expansion gives

$g(b, \phi)=g(0, \phi)+g_{b}^{\prime}(0, \phi) b+\frac{1}{2} g_{b b}^{\prime \prime}\left(b_{1}, \phi\right) b^{2}=g_{b b}^{\prime \prime}\left(b_{1}, \phi\right) b^{2}$.

where $b_{1} \in[0, b]$. It can be verified that $g(0, \phi)=0$ and $g_{b}^{\prime}(0, \phi)=0$ for all $\phi^{2}$. In addition, it holds that $g_{b b}^{\prime \prime}(0,0)=$ $\pi^{2} / 8 \omega^{6}$, which ensures $\operatorname{det}\left(A_{2}\right)>0$ for all sufficiently small $|b|$ and $|\phi|$. For the remainder of the proof, we assume that $b$ is selected suitably small to ensure $\operatorname{det}\left(A_{2}\right)>0, A_{2}$ is invertible, and the equilibrium of (13) can be found as

$$
\left(\tilde{x}_{0}^{a}, \tilde{y}_{0}^{a}\right)^{T}=-\frac{a}{2}\left(A_{2}\right)^{-1} h_{2}\left(\tilde{\theta}_{0}^{a}\right) .
$$

Taking into account that

$$
\int_{0}^{2 \pi / \omega} \int_{0}^{t} \sin (2 \omega \tau) d \tau d t=\frac{\pi}{\omega^{2}}, \quad \int_{0}^{2 \pi / \omega} \sin ^{2}(2 \omega t) d t=\frac{\pi}{\omega}
$$

the average of the equation for $\eta_{1}$ is

$$
\dot{\eta}_{1}^{a}=-\delta \omega_{1}^{\prime} \eta_{1}^{a}+\delta h_{3}\left(\tilde{\theta}^{a}, \tilde{x}^{a}, \tilde{y}^{a}\right)
$$

where

$$
\begin{aligned}
h_{3}\left(\tilde{\theta}^{a}, \tilde{x}^{a}, \tilde{y}^{a}\right)= & \omega_{1}^{\prime}\left(-k_{p}\left(\tilde{x}^{a}\right)^{2}-k_{p}\left(\tilde{y}^{a}\right)^{2}-k_{0}\left(\tilde{\theta}^{a}\right)^{2}\right)- \\
& -2 \omega_{1}^{\prime} k_{p} a\left(\tilde{x}^{a} \overline{g_{c}}+\tilde{y}^{a} \overline{g_{s}}\right)-\frac{\omega_{1}^{\prime} k_{0} b}{2 \omega^{2}} \tilde{\theta}^{a}- \\
& -\omega_{1}^{\prime} k_{p} a\left(\overline{g_{c}}+\overline{g_{s}}\right)-\frac{\omega_{1}^{\prime} k_{0} b^{2}}{8 \omega^{2}}
\end{aligned}
$$

and

$$
\overline{g_{c}}=\frac{\omega}{2 \pi} \int_{0}^{2 \pi / \omega} g_{c}(t) d t, \quad \overline{g_{s}}=\frac{\omega}{2 \pi} \int_{0}^{2 \pi / \omega} g_{s}(t) d t .
$$

The equilibrium of (16) can, therefore, be found as

$$
\tilde{\eta}_{1,0}^{a}=\frac{1}{\omega_{1}^{\prime}} h_{3}\left(\tilde{\theta}_{0}^{a}, \tilde{x}_{0}^{a}, \tilde{y}_{0}^{a}\right) .
$$

2 We used symbolic expression solver. To find $g_{b}^{\prime}(0, \phi)$ and $g_{b b}^{\prime \prime}(0,0)$ we first took the partial derivatives using the chain rule inside the integral, and then computed the integral with $b=0$. 
Finally, by averaging the last two equations of (7) and by substituting (9), (12), and (15), one gets

$$
\left(\begin{array}{c}
\dot{\eta}_{2}^{a} \\
\dot{\xi}^{a}
\end{array}\right)=\delta A_{4}\left(\begin{array}{c}
\eta_{2}^{a} \\
\tilde{\xi}^{a}
\end{array}\right)+\delta\left(\begin{array}{c}
\omega_{2}^{\prime}\left(1-k_{3}\right) h_{4}\left(\tilde{x}^{a}, \tilde{y}^{a}, \tilde{\theta}^{a}\right) \\
-\omega_{1}^{\prime} k_{3} h_{4}\left(\tilde{x}^{a}, \tilde{y}^{a}, \tilde{\theta}^{a}\right)
\end{array}\right)
$$

where

$$
\begin{gathered}
A_{4}=\left(\begin{array}{cc}
-\omega_{2}^{\prime} & -\omega_{2}^{\prime} \\
0 & -\omega_{1}^{\prime}
\end{array}\right), \\
h_{4}\left(\tilde{x}^{a}, \tilde{y}^{a}, \tilde{\theta}^{a}\right)=-k_{p}\left(\tilde{x}^{a}\right)^{2}-k_{p}\left(\tilde{y}^{a}\right)^{2}-k_{0}\left(\left(\tilde{\theta}^{a}\right)^{2}+\frac{b^{2}}{8 \omega^{2}}\right)- \\
-k_{p} a\left(2 \tilde{x}^{a} \overline{g_{c}}+2 \tilde{y}^{a} \overline{g_{s}}+a \overline{g_{c}^{2}}+a \overline{g_{s}^{2}}\right)
\end{gathered}
$$

and

$$
\overline{g_{c}^{2}}=\frac{\omega}{2 \pi} \int_{0}^{2 \pi / \omega} g_{c}(t)^{2} d t, \quad \overline{g_{s}^{2}}=\frac{\omega}{2 \pi} \int_{0}^{2 \pi / \omega} g_{s}(t)^{2} d t .
$$

Since $\operatorname{det}\left(A_{4}\right)=\omega_{1}^{\prime} \omega_{2}^{\prime}$, the unique equilibrium (18) is

$$
\left(\eta_{2,0}^{a}, \tilde{\xi}_{0}^{a}\right)^{T}=\left(A_{4}\right)^{-1}\left(\begin{array}{c}
\omega_{2}^{\prime}\left(1-k_{3}\right) h_{4}\left(\tilde{x}_{0}^{a}, \tilde{y}_{0}^{a}, \tilde{\theta}_{0}^{a}\right) \\
-\omega_{1}^{\prime} k_{3} h_{4}\left(\tilde{x}^{a}, \tilde{y}^{a}, \tilde{\theta}^{a}\right)
\end{array}\right) .
$$

Using (11) and (14), we can also conclude

$$
\left\|\left(\eta_{2,0}^{a}, \tilde{\xi}_{0}^{a}\right)\right\| \leq \frac{\max \left\{\omega_{2}^{\prime}\left(1-k_{3}\right), \omega_{1}^{\prime} k_{3}\right\} k_{0}}{8 \omega^{2}} b^{2}+O\left(a^{2}\right) .
$$

Step 2. We next seek to establish that linearization $\delta A$ of the right-hand-side of the averaged system (10), (13), (17), and (18) at the equilibrium $\left(\tilde{\theta}_{0}^{a}, \alpha_{0}^{a}, \tilde{x}_{0}^{a}, \tilde{y}_{0}^{a}, \eta_{1,0}^{a}, \eta_{2,0}^{a}, \tilde{\xi}_{0}^{a}\right)$ is a Hurwitz matrix. The matrix $A$ reads as

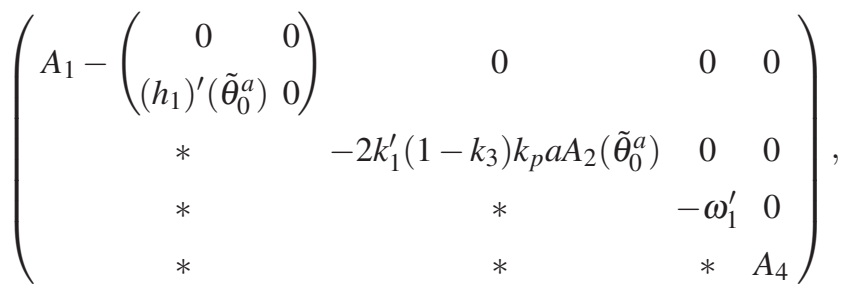

where the symbol "*" indicates for expressions, whose particular values don't influence the eigenvalues of $A$. The eigenvalues of the quasi-triangular matrix $A$ are given by the eigenvalues of the 4 diagonal blocks.

Note that a $2 \times 2$ square matrix $B$ is Hurwitz, if $\operatorname{det}(B)>0$ and $\operatorname{Tr}(B)<0$. Looking at the second diagonal block of $A$, let $f(b, \phi)$ be the trace of $A_{2}\left(\theta_{0}\right)$. Similar to the calculation of $g_{b}^{\prime}$ above, we can check that $f(0,0)=0$ and $f_{\phi}^{\prime}(0,0)=-\frac{\pi}{\omega^{2}}$. Therefore, $f(0, \phi)$ is negative (positive) for small positive (negative) value of $\phi$. In particular,

$$
\operatorname{Tr}\left(-2 k_{1}^{\prime}\left(1-k_{3}\right) k_{p} a A_{2}\left(\tilde{\theta}_{0}^{a}\right)\right)<0
$$

if $b=0$ and $\left(1-k_{3}\right) \phi$ is small and positive. Considering $b=0$, let us fix small $|\phi|$ such that $\left(1-k_{3}\right) \phi$ is positive, so that (20) holds. Then (20) will also hold for $b \in\left(0, b_{0}\right]$ provided that $b_{0}>0$ is sufficiently small. Without loss of generality we can consider $|\phi|>0$ and $b_{0}$ sufficiently small that $\operatorname{det}\left(A_{2}\right)>0$ holds for $b \in\left(0, b_{0}\right]$ as well. The second diagonal block is, therefore, Hurwitz based on the test above.

Now we address negative definiteness of the remaining diagonal blocks of $A$. We remind the reader that we did not impose any restriction on the value of $a>0$ as yet. The first diagonal block reduces to just $A_{1}$ when $a=0$ because $\left(h_{1}\right)^{\prime}\left(\tilde{\theta}_{0}^{a}\right)=0$ when $a=0$. Since $\operatorname{det}\left(A_{1}\right)=\omega_{2}^{\prime}\left(k_{2}^{\prime} k_{0}\right)(b / \omega)$ and $\operatorname{Tr}\left(A_{1}\right)=-\omega_{2}^{\prime}$ we can find $a_{0}>0$ such that the first diagonal block is Hurwitz for all $a \in\left[0, a_{0}\right]$. The third diagonal block consists of just one element $-\omega_{1}^{\prime}$ which is a Hurwitz matrix. The two eigenvalues of $A_{4}$ are $-\omega_{2}^{\prime}$ and $-\omega_{1}^{\prime}$ respectively, so that $A_{4}$ is a Hurwitz matrix as well.

To summarize, the averaged system (10), (13), (17), and (18) admits a locally exponentially stable equilibrium $e^{a}(t)=\left(\tilde{\theta}_{0}^{a}, \alpha_{0}^{a}, \tilde{x}_{0}^{a}, \tilde{y}_{0}^{a}, \eta_{1,0}^{a}, \eta_{2,0}^{a}, \tilde{\xi}_{0}^{a}\right)(t)^{T}$. The averaging principle (Thm. 10.4 in [10]) then guarantees that the original system (7) possesses a unique exponentially stable $2 \pi / \omega$-periodic solution $e(t)=\left(\tilde{\theta}, \alpha, \tilde{x}, \tilde{y}, \eta_{1}, \eta_{2}, \tilde{\xi}\right)(t)^{T}$ that verifies

$$
e^{a}(t)-e(t)=O(\delta)
$$

The required estimate (8) follows by combining (21) with the estimates (11), (14), (17), and (19).

The proof of the theorem is complete.

Remark 1. The condition of the smallness of $\phi$ can be replaced by (20), which leads to an implicit trigonometric inequality. The requirement of the smallness of $a>0$ can be replaced by implicit trigonometric inequalities that ensure stability of the equilibrium of (10). Finally, the smallness of $b>0$ can be replaced by trigonometric inequalities which guarantee that the matrix $-\left(1-k_{3}\right) A_{2}\left(\tilde{\theta}^{a}\right)$ is Hurwitz.

Remark 2. The theorem can be extended to the case where $\omega_{1}$ and $\omega_{2}$ are fixed constants (not small parameters) by applying Malkin's perturbation theory $[9,15]$ where the method of averaging is applied on a subspace.

\section{Simulation and Experiments}

\subsection{Simulation of Maximizing a Quadratic Function}

We present a simulations of the proposed ESC scheme optimizing a quadratic target function in the form of (6). We take target function as $J=10^{4}-10^{-3}\left(x-2 \cdot 10^{3}\right)^{2}-10^{-3}(y-$ $\left.3.4 \cdot 10^{3}\right)^{2}-4 \cdot 10^{3}(\theta-\pi / 4)^{2}$, and the initial coordinates of the robot are $[x, y, \theta]^{T}=[707 \mathrm{~mm}, 707 \mathrm{~mm}, \pi / 6 \mathrm{rads}]$. The dither frequency is $\omega=0.5 \pi$, the dither amplitudes are 

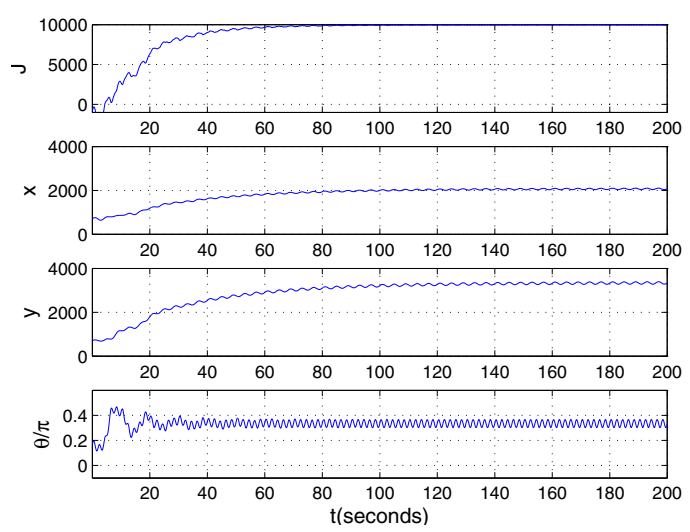

Fig. 3. Simulation Result on Quadratic Function

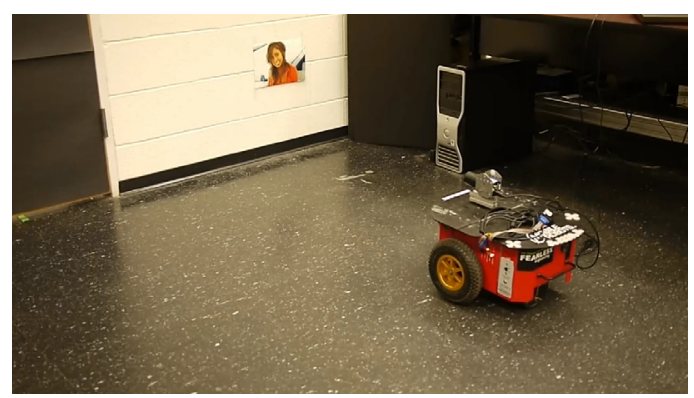

Fig. 4. Experiment Configuration

$a=100$ and $b=0.1 \pi$. The cutoff frequencies for the linear filters are $\omega_{1}=0.7 \pi$, and $\omega_{2}=0.3 \pi$. The gains are taken as $k_{1}=1, k_{2}=0.002$. The results of the simulation are shown in Fig. 3. The average value of $\theta$ quickly converges to $\theta^{*}=\pi / 4$, and the average values of $x$ and $y$ converge to $x^{*}$ and $y^{*}$ a bit slower. It is seen that the average value of the target function does reach its maximum of $10^{4}$.

\subsection{Experiment Results}

Experiments are implemented to demonstrate the performance of the proposed ESC methods in the face of common problems such as signal noise, disturbances and unmodeled system dynamics. A Pioneer -3 mobile robot is used, as shown in the Fig. 4. A poster attached to a white wall was the visual target used in the experiments. We want the robot to find the target, approach the target and settle to a good angle of view to observe the target. These goals are accomplished by maximizing a saliency metric function.

Itti's saliency model is a popular visual attention models [8]. In this model, attractiveness of visual stimuli is represented as a gray scale image called a saliency map. Regions of the image with high values will likely be of interest to a human viewing the scene. A saliency map is typically the combination of different feature maps. What features to use in the saliency model depends on the specific applications. In our experiments, we take the red-green color feature [8] to build the saliency map. The saliency metric function is generated by accumulating all the pixel values of the saliency
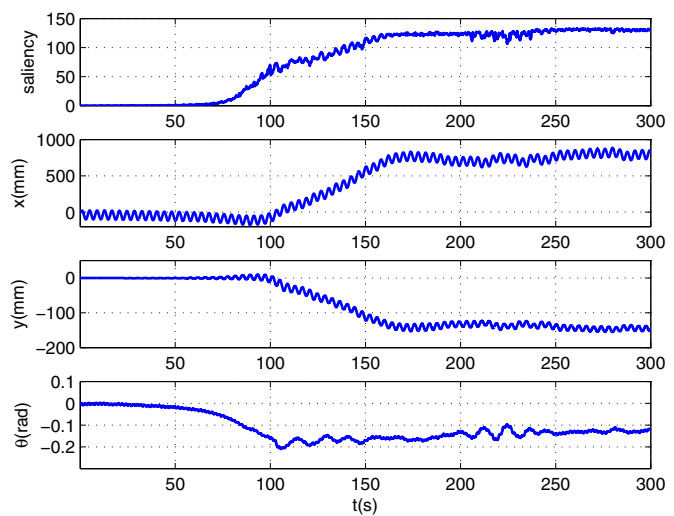

Fig. 5. The pose and saliency metric during Experiment I

map. Thus, when there are many attractive regions in an saliency map, when the pixel values of the attractive regions are very high, or when the areas of the attractive regions are large, the accumulated saliency metric will be large. The saliency maps are shown in the lower left corners in the Fig. $7 \mathrm{a}, 7 \mathrm{~b}$, and $10 \mathrm{a}-11 \mathrm{~b}$. The saliency values are shown in the lower right corners. It can be seen that the woman in the photograph is a salient region of the image, while the surrounding wall, floor, door and table have low saliency.

To ensure the target appears in the center of the image, and to prevent the robot from being too close to the target, we apply a bell-shape weighting function of image coordinates on the saliency map before calculating the saliency value.

$$
W\left(u_{p}, v_{p}\right)=\exp \left(-\left[\frac{\left(u_{p}-u_{0}\right)^{2}}{2 u_{0}^{2} \sigma_{u}^{2}}+\frac{\left(v_{p}-v_{0}\right)^{2}}{2 v_{0}^{2} \sigma_{v}^{2}}\right]\right)
$$

where $u_{p}$ and $v_{p}$ are the horizontal and vertical coordinates of the pixel $p, u_{0}, v_{0}$ and $\sigma$ are parameters to adjust the function position and shape. This function is larger when more of the image is occupied by the target, and pixels close to $\left[u_{0}, v_{0}\right]$ contribute more to the score. In our experiments, $u_{0}=512, v_{0}=384, \sigma_{u}=0.8, \sigma_{v}=0.1$.

\subsection{Experiment I}

In the first experiment, the dither frequency was $\omega=0.5 \pi$, and the dither amplitudes were $a=100, b=4$.The cutoff frequencies for the filters were $\omega_{1}=0.036$, and $\omega_{2}=0.015$. The gains were taken as $k_{1}=7000, k_{2}=0.3$. The initial position of the mobile robot was set as the origin of a planar coordinate system, i.e. $\left[x_{0}, y_{0}, \theta_{0}\right]^{T}=[0,0,0]^{T}$. The experiment data results are shown in Fig. 5. We see the saliency value keeps increasing in the top sub-figure. The bottom sub-figures show the $x, y$ coordinates and the orientation $\theta$ over time. We show the orientation and position of the robot by the pointing and position of an arrow, and show the robot trajectory by a quiver plot in Fig. 6 .

The initial scene of the robot camera is shown in Fig. 7a. In the initial stage, the effect of the weighting function dominated the saliency value, and moving the target towards the 


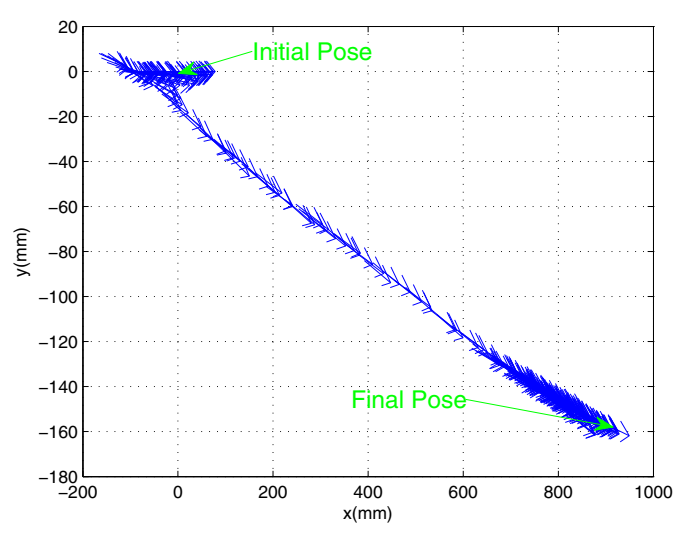

Fig. 6. The robot trajectory during Experiment I

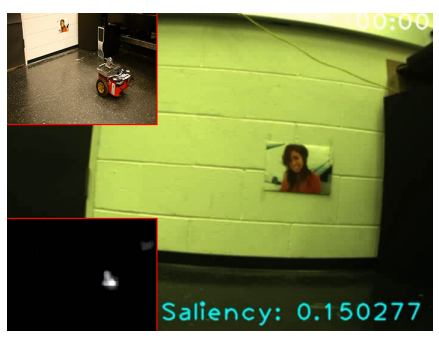

(a) The initial view

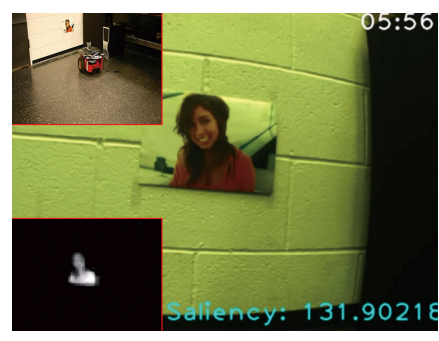

(b) The final view
Fig. 7. The views from the robot in Experiment I

center of the image increased the saliency more than moving the camera close to the target. Thus, the robot rotated to bring the target to the center of the image. The robot also moved a little bit backward in the beginning, as seen in the second top sub-figure of the Fig. 5. When the robot moves away from the target, its image takes less area on the image plane and also moves towards the image center. When using bell-shape exponential weighting function in (22), a smaller target image closer to the center may have greater saliency. After the target has been centered in the image, the robot can not increase the saliency by rotating. Thus in the following stage, we see the robot approached the target rapidly.

While the robot is moving towards the target, the target increased and moved upward. Larger image size leads to an increase in saliency, but moving upward away from the image center leads to a loss of saliency. The robot settled at a point where these factors are balanced, Fig. 7b.

\subsection{Experiment II}

The second experiment also seeks the optimal view of a target by maximizing saliency. In this experiment, we partially occluded the target to make a saliency drop after the robot had converged to a locally optimal view. This allowed us to observe how the robot responds to a sudden change of scene. We kept the dither frequency $\omega=0.5 \pi$ and the dither amplitude $a=100$, but take a larger $b=44$. The cutoff frequencies for the filters were still $\omega_{1}=0.036$, and $\omega_{2}=0.015$. The gains were taken as $k_{1}=4000, k_{2}=0.3$.The experi-
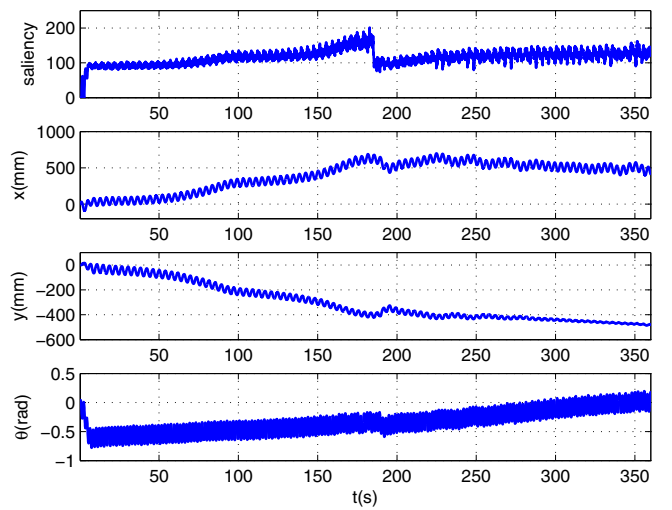

Fig. 8. The pose and saliency metric during Experiment II

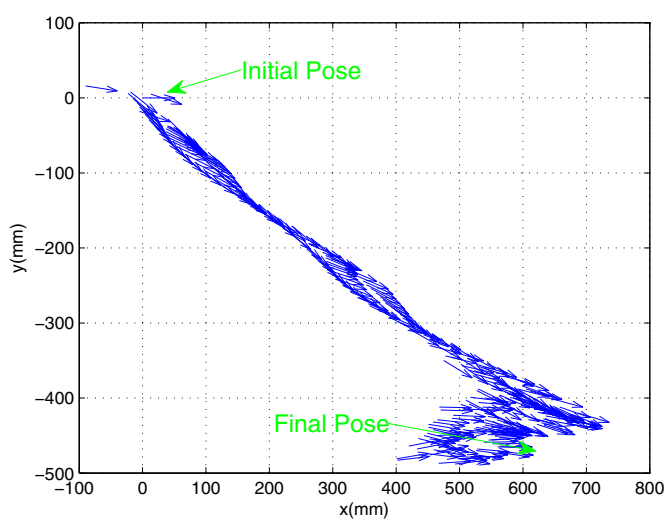

Fig. 9. The robot trajectory during Experiment II

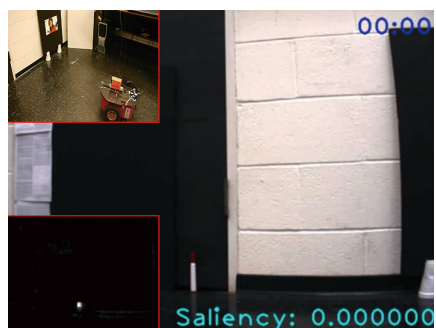

(a) The initial view

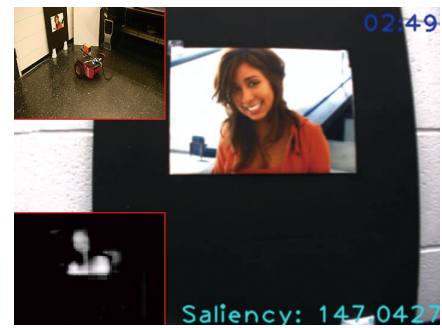

(b) Converges to a good view
Fig. 10. The views from the robot in experiment II

ment results are shown in Fig. 8, and the robot trajectory is shown in Fig. 9.

The initial scene of the robot is shown in Fig. 10a, and the target was not in the field of view at that time. Similar to the first experiment, the robot approached the target and the target moved to the center of the field of view, as shown in Fig. 10b. After the saliency value converged to a local optimal value, we partially occluded the target by a black poster board, as shown in Fig. 11a. The robot found a good observing pose again through a 'parallel parking' type motion, which was induced by the ESC scheme, Fig. 11b. Due to the characteristics of the visual system, small changes in orientation can induce large changes in the image. The 


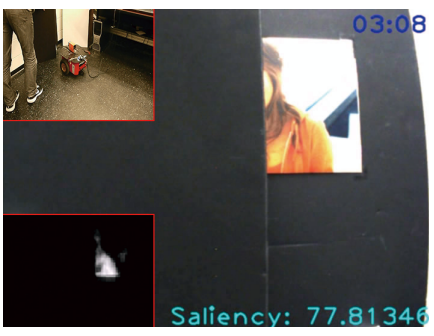

(a) Target is partially obscured.

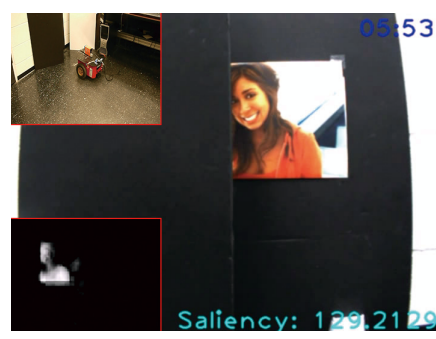

(b) Target back to view.

Fig. 11. The robot converges again after the target is blocked.

dither signal on the angular velocity induces enough orientation change that a salient target can be found in the local area even when not present in the initial FOV.

\section{Conclusion}

Many sensing tasks, such as those involving cameras, have a limited field of view, and robot orientation is critical for maximizing the target function. We have presented an ESC scheme for wheeled mobile robots with nonholonomic constraints that explicitly allows for orientation in the target function. The local exponential stability of the system was established, as was attraction of the system to a neighborhood of a maximum (or minimum for minimization problems) of the target function. We conducted simulations and experiments, including tasks where a robot must find the best viewpoint of a target. By combining a visual saliency method with a bell shape weighting function on the field of view, the robot was able to bring the target into the center of view, find an appropriate viewing distance and overcome partial occlusions. Simulation and experiment results showed the system works well. We will continue to investigate methods to increase the rate of convergence and extend the region of attraction to guarantee convergence to the global maximum.

\section{References}

[1] Jennie Cochran and Miroslav Krstic. Nonholonomic source seeking with tuning of angular velocity. IEEE Trans. on Automatic Control, 54(4):717-731, April 2009.

[2] Hans-Bernd Dürr, Miloš S Stanković, Christian Ebenbauer, and Karl Henrik Johansson. Lie bracket approximation of extremum seeking systems. Automatica, 2013.

[3] Lina Fu and Ümit Özgüner. Extremum seeking with sliding mode gradient estimation and asymptotic regulation for a class of nonlinear systems. Automatica, 47(12):2595-2603, 2011.

[4] Nicholas R. Gans and Seth A. Hutchinson. A stable vision-based control scheme for nonholonomic vehicles to keep a landmark in the field of view. In Proc. IEEE Int. Conf. on Robotics and Automation, pages 2196-2201, April 2007.

[5] Azad Ghaffari, Miroslav Krstić, and Dragan Nešić. Multivariable newton-based extremum seeking. Automatica, 48(8):1759-1767, 2012.

[6] Nima Ghods and Miroslav Krstic. Speed regulation in steering-based source seeking. Automatica, 46(2):452-459, 2010.
[7] Mark Haring, Nathan Van De Wouw, and Dragan Nešić. Extremumseeking control for nonlinear systems with periodic steady-state outputs. Automatica, 2013.

[8] L. Itti, C. Koch, and E. Niebur. A model of saliency-based visual attention for rapid scene analysis. IEEE Trans. on Pattern Analysis and Machine Intelligence, 20(11):1254 -1259, November 1998.

[9] Mikhail Kamenskii, Oleg Makarenkov, and Paolo Nistri. An alternative approach to study bifurcation from a limit cycle in periodically perturbed autonomous systems. Journal of Dynamics and Differential Equations, 23(3):425-435, 2011.

[10] H. K. Khalil. Nonlinear Systems. Prentice Hall, New Jersey, 3 edition, 2002.

[11] Sei Zhen Khong, Dragan Nešić, Ying Tan, and Chris Manzie. Unified frameworks for sampled-data extremum seeking control: Global optimisation and multi-unit systems. Automatica, 49(9):2720 - 2733, 2013.

[12] Miroslav Krstić and Hsin-Hsiung Wang. Stability of extremum seeking feedback for general nonlinear dynamic systems. Automatica, 36(4):595-601, 2000.

[13] Shu-Jun Liu and M Krstic. Stochastic nonholonomic source seeking. In Proc. IEEE Conf. on Decision and Control, pages 6985 - 6990, December 2010.

[14] Shu-Jun Liu and Miroslav Krstic. Stochastic source seeking for nonholonomic unicycle. Automatica, 46(9):1443-1453, 2010.

[15] I.G. Malkin. Some problems in the theory of nonlinear oscillations, volume 1. United States Atomic Energy Commission, Technical Information Service, Language Translation Service, Cleveland, Ohio, 1959.

[16] Alexey S Matveev, Hamid Teimoori, and Andrey V Savkin. Navigation of a unicycle-like mobile robot for environmental extremum seeking. Automatica, 47(1):85-91, 2011.

[17] William H Moase and Chris Manzie. Fast extremum-seeking for wiener-hammerstein plants. Automatica, 48(10):2433 - 2443, 2012.

[18] Dragan Nešlć, Thang Nguyen, Ying Tan, and Chris Manzie. A nongradient approach to global extremum seeking: An adaptation of the shubert algorithm. Automatica, 49(3):809-815, 2013.

[19] B. Porat and A. Nehorai. Localizing vapor-emitting sources by moving sensors. IEEE Transactions on Signal Processing, 44(4):1018 -1021, apr 1996.

[20] Mario A. Rotea. Analysis of multivariable extremum seeking algorithms. Proc. American control Conf., pages 433-437, June 2000.

[21] Miloš S Stanković and Dušan M Stipanović. Extremum seeking under stochastic noise and applications to mobile sensors. Automatica, 46(8):1243-1251, 2010.

[22] Y. Tan, D. Nesic, and I.M.Y. Mareels. On non-local stability properties of extremum seeking control. Automatica, 42, 2006.

[23] Mario Rotea Yinghua Zhang and Nicholas Gans. Sensors searching for interesting things: Extremum seeking control on entropy maps. In IEEE Conf. on Decision and Control, pages 4985-4991, 2011.

[24] Mario Rotea Yinghua Zhang, Jinglin Shen and Nicholas Gans. Robots looking for interesting things: Extremum seeking control on saliency maps. In IEEE/RSJ Int. Conf. on Intelligent Robots and Systems, pages 1180-1186, 2011.

[25] Chunlei Zhang, Daniel Arnold, Nima Ghods, Antranik Siranosian, and Miroslav Krstic. Source seeking with nonholonomic unicycle without position measurement and with tuning of forward velocity. Systems and Control Letters, 56(3):245-252, 2007.

[26] Yinghua Zhang and Nicholas Gans. Extremum seeking control of a nonholonomic mobile robot with limited field of view. In Proc. American Control Conf., June 2013. 